Portland State University

PDXScholar

6-1-1984

\title{
Energy losses of solar neutrinos and the oscillation hypothesis
}

\author{
P.T. Leung \\ Portland State University
}

S. Boedo

M. L. Rustgi

Follow this and additional works at: https://pdxscholar.library.pdx.edu/phy_fac

Part of the Physics Commons

Let us know how access to this document benefits you.

\section{Citation Details}

Leung, P. T., Boedo, S. S., \& Rustgi, M. L. (1984). Energy losses of solar neutrinos and the oscillation hypothesis. Physical Review D (Particles And Fields), 29(11), 2655-2656.

This Article is brought to you for free and open access. It has been accepted for inclusion in Physics Faculty Publications and Presentations by an authorized administrator of PDXScholar. Please contact us if we can make this document more accessible: pdxscholar@pdx.edu. 


\title{
Energy losses of solar neutrinos and the oscillation hypothesis
}

\author{
P. T. Leung \\ Physics Department, Tamkang University, Tamsui, Taiwan 251, Republic of China \\ S. Boedo* and M. L. Rustgi \\ Physics Department, State University of New York, Buffalo, New York 14260
}

(Received 12 December 1983)

\begin{abstract}
A formula for the stopping power of neutrinos interacting via the standard weak-interaction model, but incorporating the possibility of neutrino oscillations among the three flavors, is derived. The results are applied to study the solar-neutrino anomaly and it is found that the anomaly cannot be accounted for by many orders of magnitude from consideration of the energy losses of the neutrinos interacting with the solar matter, even if the oscillation hypothesis is found to be valid.
\end{abstract}

Ever since the experiment performed by Davis and his collaborators $^{1}$ and the discrepancy observed between the experimental data and the theoretical results calculated based on the standard solar model, the solar-neutrino anomaly has puzzled theorists and experimentalists. In two previous publications, ${ }^{2,3}$ the possibility of explaining the anomaly from consideration of the energy losses of the neutrinos interacting with the electrons in the solar plasma has been investigated. In particular, by considering the possible electromagnetic and weak interactions between the neutrinos and the electrons, it had been concluded that the anomaly is not likely to be accounted for from considerations of the energy losses of the solar neutrinos. In order to have a more complete conclusion, in this note we reinvestigate the prob- lem of the energy losses taking into account the possibility of oscillations of the neutrino among its three flavors: $\nu_{e}$, $\nu_{\mu}$, and $\nu_{\tau}$.

Because of the possibility of the mixing of the neutrino mass eigenstates and weak eigenstates in the standard Weinberg-Salam model, a neutrino can oscillate among the three flavors $\nu_{e}, \nu_{\mu}$, and $\nu_{\tau}$ (corresponding to the three known leptons $e, \mu$, and $\tau$ ) as it travels in space. ${ }^{4}$ Taking into consideration this oscillation process, we shall work out the $\nu_{e}-e$ scattering cross section. For antineutrinos, the $\bar{\nu}_{e}-e$ scattering process has been worked out by Halls and McKellar, ${ }^{5}$ in terms of the recoil energy $Q$ of the electron; the result is ${ }^{6}$

$$
\begin{aligned}
\frac{d \sigma}{d Q}=\frac{G^{2} m_{e}}{2 \pi} & \left\{P_{e}(t)\left[4\left(1-\frac{Q}{E_{\nu}}\right)^{2}+4\left(g_{A}+g_{V}\right)\left(1-\frac{Q}{E_{\nu}}\right)^{2}+\left(g_{A}-g_{V}\right) \frac{m_{e} Q}{2 E_{\nu}{ }^{2}}\right]\right. \\
& \left.+\left(g_{A}+g_{V}\right)^{2}\left(1-\frac{Q}{E_{\nu}}\right)^{2}+\left(g_{A}-g_{V}\right)^{2}+\frac{m_{e} Q}{E_{\nu}{ }^{2}}\left(g_{A}{ }^{2}-g_{V}{ }^{2}\right)\right\},
\end{aligned}
$$

where $m_{e}$ is the mass of the electron, $E_{\nu}$ is the energy of the incident neutrino, and

$$
\frac{G^{2} m_{e}}{2 \pi}=4.1 \times 10^{-45} \mathrm{~cm}^{2} / \mathrm{MeV}, \quad g_{V}=-\frac{1}{2}+2 \sin ^{2} \theta_{W}, \quad g_{A}=-\frac{1}{2} .
$$

The quantity $P_{e}(t)$ in Eq. (1) is the probability of the electron antineutrino to preserve its identity at a time $t$ satisfying the relation

$$
P_{e}(t)+P_{\mu}(t)+P_{\tau}(t)=1
$$

Though the results quoted in Ref. 5 indicate the insensitivity of the energy loss in the sun of solar neutrinos to $\nu_{e}, \nu_{\mu}$, and $\nu_{\tau}$ oscillations, the $\nu_{e}-e$ scattering cross section cannot be obtained simply by replacing $g_{A}$ by $-g_{A}$ in Eq. (1), since the scattering occurs via a mixture of charged and neutral currents for the $\nu_{e}-e$ (Ref. 7) process, while only the neutral current contributes to the $\nu_{\mu}-e$ and ${ }^{8} \nu_{\tau}-e$ scattering processes. To do this, we have to go back to the original formalism with the constants $C_{L}$ and $C_{R}$ associated with the left and the right electron currents, respectively. ${ }^{9}$ The result reexpressed in terms of the $g_{A}$ and $g_{V}$ in Eq. (2) is

$$
\frac{d \sigma}{d Q}=\frac{G^{2} m_{e}}{2 \pi}\left[P_{e}(t)\left(4\left(g_{A}+g_{V}+1\right)+2\left(g_{A}-g_{V}\right) \frac{m_{e} Q}{E_{\nu}{ }^{2}}\right)+\left(g_{A}+g_{V}\right)^{2}+\left(g_{A}-g_{V}\right)^{2}\left(1-\frac{Q}{E_{\nu}}\right)^{2}+\frac{m_{e} Q}{E_{\nu}{ }^{2}}\left(g_{A}{ }^{2}-g_{V}{ }^{2}\right)\right] .
$$

It should be remarked that in the case when $P_{e}(t)=1$, i.e., no oscillation occurs, both Eqs. (1) and (4) give back the wellknown results due first to 't Hooft and others. ${ }^{10}$

To apply Eq. (4) to study the energy loss of solar neutrinos, we compute the stopping power for neutrinos in the high- $Q$ limit treating the solar electrons to be free. Following the theory of energy losses, ${ }^{11,12}$ we obtain 
TABLE I. Stopping power and energy loss for solar neutrinos for two values of $P_{e}$

\begin{tabular}{|c|c|c|c|c|c|}
\hline \multirow[b]{2}{*}{$E_{\nu}(\mathrm{MeV})$} & \multirow[b]{2}{*}{$(\mathrm{MeV})$} & \multicolumn{2}{|c|}{$P_{e}=0.4$} & \multicolumn{2}{|c|}{$P_{e}=1.0$} \\
\hline & & $-\frac{d E}{d S}(\mathrm{MeV} / \mathrm{cm})$ & $-\Delta E(\mathrm{MeV})$ & $-\frac{d E}{d S}(\mathrm{MeV} / \mathrm{cm})$ & $-\Delta E(\mathrm{MeV})$ \\
\hline 1 & 0.80 & $1.6 \times 10^{-20}$ & $1.1 \times 10^{-9}$ & $3.1 \times 10^{-20}$ & $2.18 \times 10^{-9}$ \\
\hline 2 & 1.77 & $8.1 \times 10^{-20}$ & $5.7 \times 10^{-9}$ & $15.7 \times 10^{-20}$ & $11.0 \times 10^{-9}$ \\
\hline 3 & 2.77 & $19.7 \times 10^{-20}$ & $13.8 \times 10^{-9}$ & $39.1 \times 10^{-20}$ & $27.3 \times 10^{-9}$ \\
\hline 5 & 4.76 & $58.5 \times 10^{-20}$ & $41.0 \times 10^{-9}$ & $116.6 \times 10^{-20}$ & $81.6 \times 10^{-9}$ \\
\hline 8 & 7.75 & $155.6 \times 10^{-20}$ & $108.9 \times 10^{-9}$ & $311.2 \times 10^{-20}$ & $217.8 \times 10^{-9}$ \\
\hline 10 & 9.75 & $246.3 \times 10^{-20}$ & $172.4 \times 10^{-9}$ & $493.6 \times 10^{-20}$ & $345.5 \times 10^{-9}$ \\
\hline
\end{tabular}

$$
\begin{aligned}
\frac{-d E}{d S}=\frac{N G^{2} m_{e}}{2 \pi}\{ & {\left[2 P_{e}\left(g_{A}+g_{V}+1\right)+\left(g_{A}{ }^{2}+g_{V}{ }^{2}\right)\right]\left(Q_{m}{ }^{2}-\eta^{2}\right) } \\
& \left.-\left(\frac{2}{E_{\nu}}\left(g_{A}-g_{V}\right)^{2}-\left(2 P_{e}+g_{A}+g_{V}\right)\left(g_{A}-g_{V}\right) \frac{m_{e}}{E_{\nu}{ }^{2}}\right)\left(\frac{Q_{m}{ }^{3}-\eta^{3}}{3}\right)+\frac{1}{E_{\nu}{ }^{2}}\left(g_{A}-g_{V}\right)^{2}\left(\frac{Q_{m}{ }^{4}-\eta^{4}}{4}\right)\right\},
\end{aligned}
$$

where $N$ is the number density of electrons in the medium, $Q_{m}=2 E_{\nu}{ }^{2} /\left(m_{e}+2 E_{\nu}\right)$ is the maximum energy transferred to the electron, and $\eta$ is approximately given by the plasmon energy of the solar electrons. Similar results can be obtained for the energy losses of antineutrinos by employing Eq. (1). Due to moderate energies of and enormous distances traveled by solar neutrinos, $P_{e}$ has been estimated to lie within the range ${ }^{13}$

$$
0.39 \leqslant P_{e} \leqslant 0.86
$$

For solar electrons, $N=1.2 \times 10^{25} \mathrm{~cm}^{-3}$ and $\eta \sim \hbar\left(4 \pi N e^{2} /\right.$ $\left.m_{e}\right)^{1 / 2} \sim 100 \mathrm{eV}$. With all these numerical values and taking $\sin ^{2} \theta_{W}=0.224$ in (2), we have computed Eq. (5) for two different values of $P_{e}, P_{e}=0.4$ and $P_{e}=1$, corresponding to no oscillations. Furthermore, if we assume that this stopping power is constant all along the range of the neutrino, then the total energy loss $(-\Delta E)$ for a neutrino created at the center and escaping at the surface of the sun can be calculated just by multiplying the stopping power with the radius of the sun $-7 \times 10^{10} \mathrm{~cm}$. The numerical results are shown in Table I for different energies of the solar neutrinos.

The decrease in energy loss with decrease in $P_{e}$ can be seen if one collects all the terms involving $P_{e}$ in Eq. (5) and employs Eq. (2). One obtains

$$
4 P_{e} \sin ^{2} \theta_{W}\left(1-\frac{2}{3\left(1+2 E_{v} / m_{e}\right)}\right),
$$

where use has been made of the fact that for all the neutrino energies considered $\eta<<Q_{m}$. It is obvious that (7) decreases as $P_{e}$ decreases implying a decrease in $(-d E / d S)$.

From the results, we see that the energy losses of the solar neutrinos are of the same order of magnitude when compared with those values when no oscillation occurs. ${ }^{3}$ The exact numerical values are even smaller in this case. The energy lost by $1-\mathrm{MeV}$ neutrinos traversing through the sun is $\sim 10^{-9} \mathrm{MeV}$ and that for $10-\mathrm{MeV}$ neutrinos is $\sim 10^{-7} \mathrm{MeV}$, which are completely insignificant. Thus, together with the two previous investigations, ${ }^{2,3}$ we can conclude more safely and completely that the solar-neutrino anomaly is not likely to be accounted for from considerations of energy losses of the neutrinos due to interaction with the solar matter as it escapes through the sun.

One of the authors (M.L.R.) is grateful to Dr. Richard Gonsalves for his help in locating one of the experimental references. This work was partially supported by the National Aeronautics and Space Administration under Grant No. NAG1442.
*Now at the Department of Theoretical and Applied Mechanics, Cornell University, Ithaca, New York 14853.

${ }^{1}$ See, e.g., R. Davis, Jr., Phys. Rev. Lett. 12, 303 (1964); B. Kuchowicz, Rep. Prog. Phys. 39, 21 (1976); J. Bernstein, M. Ruderman, and G. Feinberg, Phys. Rev. 132, 1227 (1963); J. N. Bahcall et al., Rev. Mod. Phys. 54, 767 (1982).

${ }^{2}$ M. L. Rustgi, P. T. Leung, J. E. Turner, and W. B. Brandt, Phys. Rev. A 24 , 2425 (1981); 25,2417 (1982).

${ }^{3}$ P. T. Leung and M. L. Rustgi, Phys. Rev. A 25, 2588 (1982).

${ }^{4}$ See, e.g., A. DeRújula, M. Lusignoli, L. Maiani, S. T. Petcov, and R. Petronzio, Nucl. Phys. B168, 54 (1980); V. Barger, K. Whisnant, D. Cline, and R. J. N. Phillips, Phys. Lett. 93B, 194 (1980); J. N. Bahcall, S. H. Lubow, W. F. Huebner, N. H. Magee, Jr., A. L. Merts, M. F. Argo, P. D. Parker, B. Rozsnyai, and R. K. Ulrich, Phys. Rev. Lett. 45, 945 (1980); V. Barger, K. Whisnant, and R. J. N. Phillips, Phys. Rev. D 24, 538 (1981).

${ }^{5}$ B. Halls and B. H. J. McKellar, Phys. Rev. D $\underline{24}, 1785$ (1981).

${ }^{6} \mathrm{We}$ believe that there is an error in the result obtained by Halls and McKellar; our Eq. (1) should be compared and contrasted with Eq. (4) of Ref. 5.

${ }^{7}$ For an experimental detection of the only reaction in which both $W^{-}$and $Z^{0}$ are involved, see F. Reines, H. S. Gurr, and H. W. Sobel, Phys. Rev. Lett. 37, 315 (1976).

${ }^{8}$ For the experimental observation of $\nu_{\mu}+e^{-} \rightarrow \nu_{\mu}+e^{-}$, see L. A. Ahrens et al., Phys. Rev. Lett. 51, 1514 (1983). This paper contains a detailed bibliography of the earlier publications.

${ }^{9}$ See, e.g., J. C. Taylor, Gauge Theories of Weak Interactions (Cambridge University, Cambridge, England, 1974), pp. 57-61.

${ }^{10} \mathrm{G}$. 't Hooft, Phys. Lett. 37B, 195 (1971); also see, e.g., H. H. Chen and B. W. Lee, Phys Rev. D $\underline{5}, 1874$ (1972); L. M. Sehgal, Nucl. Phys. B70, 61 (1974).

${ }^{11} \mathrm{H}$. A. Bethe, in Handbuch der Physik, Vol. 24, edited by H. Geiger and K. Scheel (Springer, Berlin, 1933), pp. 491ff.

${ }^{12}$ U. Fano, Annu. Rev. Nucl. Sci. 13, 1 (1963).

${ }^{13}$ See De Rújula et al. (Ref. 4). 